\title{
Photo-Electrical RESPONSE OF DONOR- ACCEPTOR COMPLEX WITH CARBONYL VIOLOGEN ACCEPTOR
}

\author{
Nikola Kirov ${ }^{1}$, Tsvetanka Philipova ${ }^{2}$, Milka Hadjieva ${ }^{3}$, Katya Ivanova ${ }^{3}$, Ivo \\ Grabchev ${ }^{4}$ and Georgi B. Hadjichristov ${ }^{1, *}$ \\ ${ }^{1}$ Laboratory of Optics and Spectroscopy, Institute of Solid State Physics, Bulgarian \\ Academy of Sciences, Sofia, Bulgaria \\ ${ }^{2}$ Department of Organic Chemistry, University of Chemical Technology and \\ Metallurgy, Sofia, Bulgaria \\ ${ }^{3}$ Central Laboratory of Solar Energy and New Energy Sources, Bulgarian Academy of \\ Sciences, Sofia, Bulgaria \\ ${ }^{4}$ Institute of Polymers, Bulgarian Academy of Sciences, Sofia, Bulgaria \\ georgibheissp.bas.bg
}

\begin{abstract}
The photo-electrical response of donor-acceptor complex formed by carbonyl viologen 1,1'-bis[2-(4methoxyphenyl)-2-oxoethyl]-4,4'-bipyridine-1,1'-diium bromide (as electron acceptor), and 1Aminonaphthalene (electron donor), is studied in acetonitrile solutions under illumination close to solar irradiation conditions. The effect of the donor-to-acceptor molar ratio on the photo-electrical properties of the molecular complex is established. The molecular system containing carbonyl viologen acceptor exhibits an efficient photo-electrical response that may be of interest for solar energy conversion.
\end{abstract}

\section{KEYWORDS}

Viologen, Photoresponsive donor-acceptor complexes, Solar light conversion

\section{INTRODUCTION}

The electron Donor-Acceptor (DA) molecular systems based on the reversible electron-transfer (ET) photochemistry of viologen copounds [1-4] have attracted a great deal of study by diverse groups of researchers due to their potential for preparation of materials for light conversion and other applications [3,5-8]. Of special interest for the technology of solar energy conversion are the photoelectro-chemical ETDA systems, either in solutions and solid state, which are photoactive in the visible. Upon visible-light illumination, such ETDA complexes containing viologen (as an electron acceptor) and electron-donating molecule are able to absorb a considerable amount of energy, that results in ET and in a formation of photo-generated free radicals (Figure 1a) [9-11]. A recombination of the $\mathrm{D}-\mathrm{A}$ radical ion pair $\left(\mathrm{D}^{+} / \mathrm{A}^{-}\right)$and spontaneous ET can turn the system back to its ground state. Both processes are related to the well known reversible intramolecular ET mechanism [9] controlled by the viologen molecule [3,4] (Figure 1a).

Photophysical properties of 1,1'-disubstituted 4,4'-bipyridinium viologens modified by inclusion of carbonyl groups in their molecular structure have been reported [12]. These compounds were synthesized to be employed as electron acceptors in photochemical ETDA systems for solar light conversion applications (combined solar energy conversion and storage). In the present study, we have focused our attention on the photo-electrical properties of ETDA 
molecular system based on the carbonyl-containing viologen 1,1'-bis[2-(4-methoxyphenyl)-2oxoethyl]-4,4'-bipyridine-1,1'-diium bromide, used as an electron acceptor. Electron donor in the ETDA was $\alpha$-naphthylamine (IUPAC name: 1-Aminonaphthalene). Owing to the amino groups, the aromatic amines possess strong electron-donating ability, and the electron DA systems constituted from viologens and amines exhibit an efficient and reversible ET [13].

a)

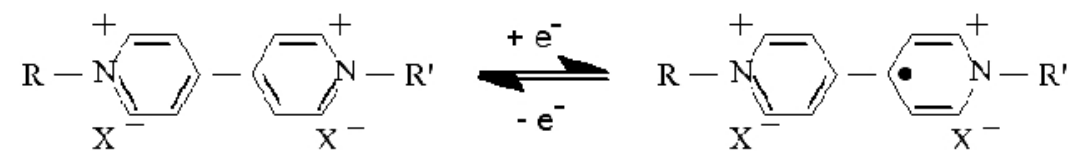

b)

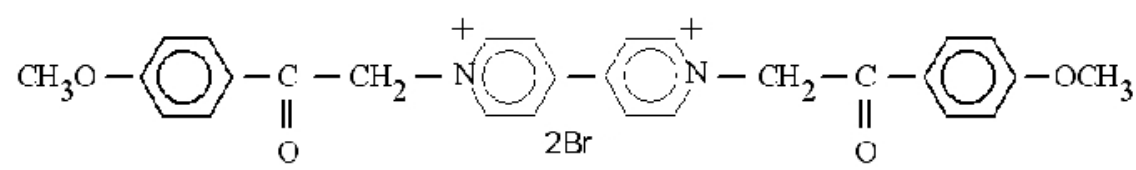

Figure 1. a) Scheme of reversible electron-transfer (ET) mechanism controlled by the viologen molecule; (b) Chemical structure of the molecule of carbonyl-containing viologen 1,1'-bis[2-(4-methoxyphenyl)-2-oxoethyl]-4,4'-bipyridine-1,1'-diium bromide.

\section{EXPERIMENTAL}

The chemical structure of the molecule of carbonyl-containing viologen 1,1'-bis[2-(4methoxyphenyl)-2-oxoethyl]-4,4'-bipyridine-1,1'-diium bromide is shown in Figure $1 b$. The synthesis and characterization of this modified viologen have been reported elsewhere [12]. The viologen exhibits photophysical properties which are very attractive for light-conversion applications. Further, the compound is stable and highly soluble in water and acetonitrile. Moreover, the viologen is stable upon solar irradiation conditions.

Photoresponsive viologen-containing DA molecular systems were formed as liquid solutions, by mixing of a solution of the viologen with a solution of the electron-donating 1Aminonaphthalene (from Aldrich) at three Donor-to-Acceptor (D:A) molar ratios, namely 1:2, 1:1 and 2:1. The solvent was aqueous acetonitrile (a mixture of acetonitrile and distilled water in a volume/volume ratio of 9:1). The effect of this highly polar solvent on the efficiency of ET process in viologen/aminoaromatics DA solutions is well established [11,13].

The photo-electrical response of the DA solutions was studied by electrical conductance measurements. For purpose, the solutions were introduced in assembly with two platinum electrodes. The ac conductance of the solution between the electrodes was determined by measuring the complex impedance using a digital LCR meter (HP, Model 4274A). A small voltage with amplitude of $2 \mathrm{mV}$ at frequency of $100 \mathrm{kHz}$ was applied on the electrodes. The measurement instrument was accurate to $\pm 0.1 \%$. In order to be examined under irradiation conditions close to those of solar irradiation, the DA systems were illuminated with a white light. As a light source, a continuous $25 \mathrm{~W}$ tungsten filament lamp was employed. The light from the lamp was passing through a Schott Glass filter GG495 (2.5 mm thickness, colloidallycolored glass; long-pass filter; cut-off at $500 \mathrm{~nm}$ ). The steady illumination was done for periods of ten minutes alternated with dark periods.

The optical absorption spectra of solutions of both donor and acceptor components, as well as the one of the DA complex, were recorded over the wavelength range $250-800 \mathrm{~nm}$ using a 
Jobin-Yvon JY3D spectrophotometer. The solutions were measured in quartz cuvettes with 1 $\mathrm{mm}$ optical path length. All experiments were carried out at room temperature (kept fixed at 22 $\left.{ }^{\circ} \mathrm{C}\right)$.

\section{RESULTS AND DISCUSSION}

As known, the introduction of the polar carbonyl $(\mathrm{C}=\mathrm{O})$ groups in the structure of the viologen molecule gives rise to the charge separation and strongly contributes to the stabilization of viologen in polar media [14]. Moreover, one can expect that the electron-attracting properties of carbonyl group should enhance the electron affinity of the viologen.

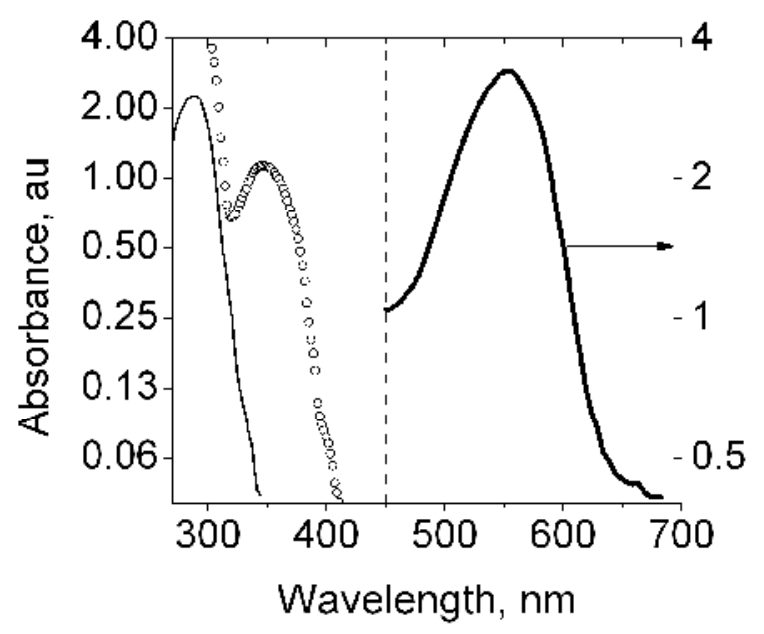

Figure 2. Absorption spectra of carbonyl viologen (thin line) and 1-Aminonaphthalene (circles). The absorption of DA charge-transfer complex formed by mixing the viologen and 1-Aminonaphthalene is shown with bold line.

Figure 2 reports the absorption spectra of the carbonyl viologen (dissolved at concentration of $10^{-3} \mathrm{M}$ in aqueous acetonitrile) and 1-Aminonaphthalene (at concentration of $2 \times 10^{-2} \mathrm{M}$ in acetonitrile), as well as the absorption band of their mixture. As seen, the latter is centered at $555 \mathrm{~nm}$, i.e. red-shifted at least by $100 \mathrm{~nm}$ from the electronic absorption bands of the two nonmixed (and uncomplexed) components. The presence of this band means that a molecular complex is successfully formed upon mixing the carbonyl viologen and 1-Aminonaphthalene. From a practical point of view, of interest is mainly the fact that the absorption band of the formed molecular system is within the region of maximum solar intensity, i.e. in the wavelength range of yellow-green light $(500-600 \mathrm{~nm})$. The new electronic band arising is assigned to the charge-transfer (CT) band of the complex.

The photoactive state of the complex is associated with the CT state (the excited state from which the ET occurs). By photo-excitation of this state one can examine the complex as a photoresponsive system. In our case, of interest is the photo-electrical response of the complex upon repeated solar illumination. Figure 3 presents the time course of the photo-induced electrical conductance measured for the studied molecular complex (the D-A pair 1Aminonaphthalene/viologen) in solution upon the alternation of illumination and dark. The photocurrent is indicative of the photo-induced charge separation and the reversibility of the electronic transition of the complex. This measurement clearly indicates a relatively fast and, what is more important, reversible photo-electrical effect. 
Further, one can compare the photo-electrical properties of the ETDA complex in solutions by varying the D:A molar ratio, keeping the other experimental conditions identical. In Figure 3 are shown the electrical conductance measured for the complex at three D:A ratios (1:2, 1:1 and 2:1). The effect of D:A molar ratio can be estimated through the level of the photo-induced conductance and other energy-conversion characteristics of the measured ETDA complex. From the photo-electrical measurements one can evaluate the characteristics of photo-electrical response of the examined molecular systems, such as the stationary value of conductance $\left(\sigma_{0}\right)$ responsible for a complete energy-transfer process. Of practical interest are also the rate constants (the increase rate constant, $k_{1}$, and the decay rate constant, $k_{2}$ ).

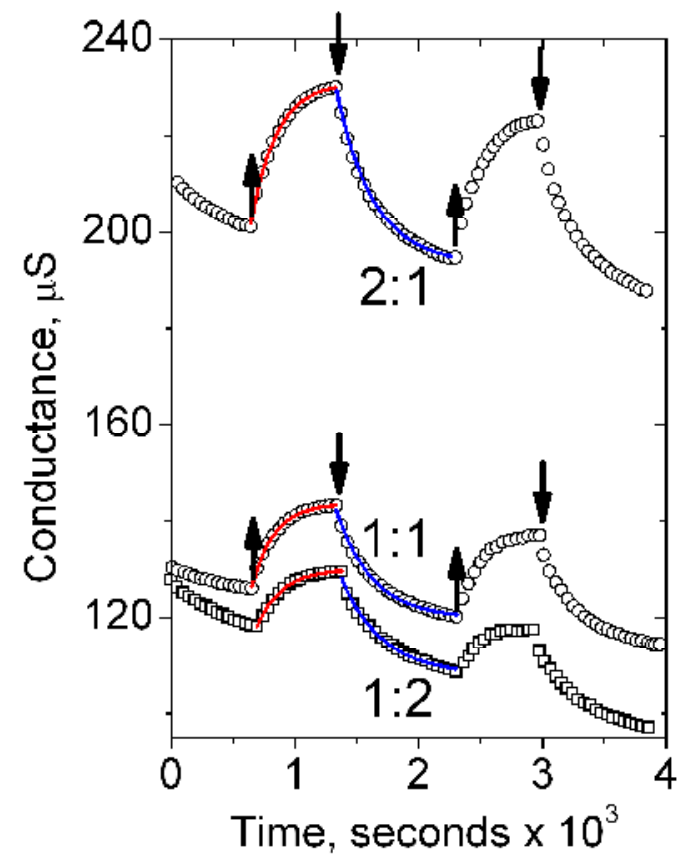

Figure 3. Electrical conductance versus time as measured for electron DA complexes formed by 1-Aminonaphthalene and carbonyl viologen at various D:A molar ratio: 1:2, 1:1

and 2:1. The illumination (white light, $\lambda>500 \mathrm{~nm}$ ) is periodically switched-on and switched-off, as indicated with the up-arrows and down-arrows, respectively. The lines represent the fits to the experimental data (the fits by exponential functions according to Eq. 1 (red lines) and Eq. 2 (blue lines)).

In our case, the illumination results in a relatively steady photocurrent with no noise. The rise and decay of the photocurrent are associated with charge carriers generation and charge recombination of the $\mathrm{D}-\mathrm{A}$ radical ion pair $\mathrm{D}^{+} / \mathrm{A}^{-}$, respectively. The experimentally obtained temporal variation of both rise and decay of the photocurrent of the measured molecular systems can be exactly fitted by a simple mono-exponential function. Thus, these behaviors for the electrical conductance $(\sigma)$ were fitted by the following functions:

$$
\begin{aligned}
& \sigma=\sigma_{0}\left[1-A^{*} \exp \left(-0.001 k_{1} t\right)\right] \\
& \sigma=B^{*} \exp \left(-0.001 k_{2} t\right)+C,
\end{aligned}
$$

for the rise and decay parts, respectively, where $A, B$ and $C$ are constants, $t$ denotes the time variable, and 0.001 is a scale factor. Fits are given in Figure 3. 
Figure 4 shows the results from the fitting of the experimental data presented in Figure 3. As seen from the values of $\sigma_{0}$, the photo-electrical response of the ETDA systems can be considerably changed by the D:A molar ratio. The optimal photo-electrical response renders the high $\sigma_{0}$ and low values for the rate constants. In our case, the experimental data and calculated characteristics indicate that the photo-excitation leads to a charge carriers generation whose efficiency increases with the increasing D:A molar ratio. Accordingly, the performance of the ETDA system is also improved.

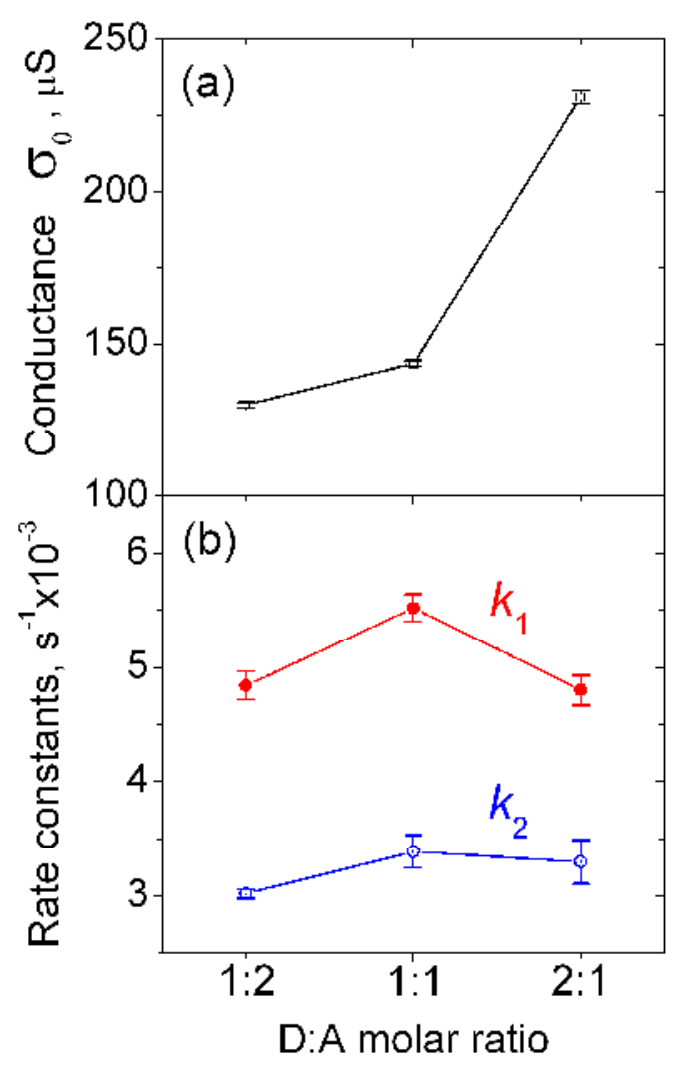

Figure 4. Plots of the experimentally assessed parameters $\sigma_{0}$ (a) and $\mathrm{k}_{1}$ and $\mathrm{k}_{2}$ (b). The conductance $\sigma_{0}$ and rate constants $\mathrm{k}_{1}$ and $\mathrm{k}_{2}$ found by fitting the experimental data from

Figure 3. The error bars represent the uncertainty of the fits.

The dynamic balance between the carrier generation and recombination indicates that after turning off the illumination, the photocurrent due to spontaneous reverse process (the charge recombination of the D-A radical ion pair) declines with a relaxation time that is longer as compared to the rise time of the photocurrent upon illumination. Thus, the DA complex under study has a potential for a photo-sensitized energy-transfer cycle, and solar energy conversion, respectively.

\section{Conclusions}

The donor-acceptor complex (in aqueous acetonitrile solution) of 1-Aminonaphthalene (the electron donor) and the viologen compound 1,1'-bis[2-(4-methoxyphenyl)-2-oxoethyl]-4,4'bipyridine-1,1'-diium bromide (the acceptor) exhibits a clearly expressed photo-electrical response upon visible-light illumination. The photocurrent has a relatively slow response to the illumination switching. As expected, the donor-to-acceptor molar ratio affects the photo-induced 
energy transfer and photo-electrical properties of the examined ETDA molecular system. Our experiment shows a correlation between the increase of this ratio and the improvement of the photo-electrical properties of ETDA complex formed by use of the carbonyl-containing viologen compound we have studied. Since the photo-excitation occurs with light in the region of maximum solar intensity, such ETDA complexes may be useful for photochemical solar cells, if the performance of the photoactive molecular system will be improved. Furthermore, ETDA complexes with carbonyl viologen acceptor can be applied in molecular components and devices utilizing the photo-induced electron and energy transfer, that is the typical application of viologens in molecular electronics.

\section{REFERENCES}

[1] L.A. Summers (1984) “The bipyridines”, Adv. Heterocyc. Chem., Vol. 35, pp 281-394.

[2] P. M. S. Monk \& N. M. Hodgkinson (1998), "Charge-transfer complexes of the viologens : effects of complexation and the rate of electron transfer to methyl viologen", Electrtrocim. Acta, Vol. 43, No. 3-4, pp 245-255.

[3] P. M. S. Monk (1998) The Viologens: Physicochemical Properties, Synthesis and Applications of the Salts of 4,4'-Bipyridine, Chichester, John Wiley \& Sons.

[4] M. Nanasawa (1999) "Photochromism by electron transfer: photochromic viologens", Organic Photochromic and Thermochromic Compounds, Topics in Applied Chemistry, Vol. 1, Main Photochromic Families (eds. J. C. Crano \& R. J. Guglielmetti), New York, Plenum Press, pp 341-370.

[5] J. Rabani (1990) "Polyelectrolytes assessment of their possible role in future solar energy storage", Photochemical Conversion and Storage of Solar Energy (eds. E. Pelizzetti \& M. Schiavello), Berlin, Springer-Verlag, pp 103-120.

[6] P. M. S. Monk, R. J. Mortimer \& D. R. Rosseinsky (1995) Electrochromism: Fundamentals and Applications, Weinheim (Germany), VCH, pp 124-142.

[7] R. J. Mortimer, A. L. Dyer \& J. R. Reynolds (2006) "Electrochromic organic and polymeric materials for display applications", Displays, Vol. 27, No. 1, pp 2-18.

[8] P. M. S. Monk, R. J. Mortimer \& D. R. Rosseinsky (2007) Electrochromism and Electrochromic Devices, Cambridge (UK), Cambridge University Press, pp 341-373.

[9] J. S. Connoly \& J. R. Bolton (1988) "Intramolecular electron transfer: hystory and some implications for artificial photosynthesis", Photoinduced Electron Transfer (eds. M. A. Fox \& M. Chanon), Amsterdam, Elsevier, part D, pp 303-393.

[10] R. S. Mulliken \& W. B. Person (1969) Molecular Complexes: A Lecture and Reprint Volume, New York, Wiley-Interscience.

[11] Y. Nishimura, H. Misawa, H. Sakuragi \& K. Tokumaru (1989) "Effects of organic solvents on charge separation in photo-induced electron transfer from xanthene dyes to methyl viologen”,Chem. Lett., Vol. 18, No. 9, pp 1555-1558.

[12] N. Kirov, Ts. Philipova, M. Hadjieva, K. Ivanova \& G. B. Hadjichristov (2009) "Photophysical properties of modified viologens for light conversion”, Compt. Rend. Acad. Bulg. Sci., Vol. 62, No. 4, pp 433-438.

[13] A. T. Poulos, C. K. Kelley \& R. Simone (1981) "Photoinduced electron transfer in the pphenylenediamine-paraquat molecular complex", J. Phys. Chem., Vol. 85, No. 6, pp 823-828.

[14] V. Ya. Shafirovich, E. E. Batova \& P. P. Levin (1991) "Unidirectional electron transfer in the supramolecules: the control by an external magnetic field", Photochemical Conversion and Storage of Solar Energy: Proceedings of the Eighth International Conference on Photochemical Conversion and Storage of Solar Energy (eds. E. Pelizzetti \& M. Schiavello), Dordrecht, Kluwer, pp 47-66. 